\title{
Functional requirements driving the gene duplication in 12 Drosophila species
}

\author{
Yan Zhong ${ }^{\dagger}$, Yanxiao Jia ${ }^{\dagger}$, Yang Gao, Dacheng Tian, Sihai Yang ${ }^{*}$ and Xiaohui Zhang ${ }^{*}$
}

\begin{abstract}
Background: Gene duplication supplies the raw materials for novel gene functions and many gene families arisen from duplication experience adaptive evolution. Most studies of young duplicates have focused on mammals, especially humans, whereas reports describing their genome-wide evolutionary patterns across the closely related Drosophila species are rare. The sequenced 12 Drosophila genomes provide the opportunity to address this issue.

Results: In our study, 3,647 young duplicate gene families were identified across the 12 Drosophila species and three types of expansions, species-specific, lineage-specific and complex expansions, were detected in these gene families. Our data showed that the species-specific young duplicate genes predominated (86.6\%) over the other two types. Interestingly, many independent species-specific expansions in the same gene family have been observed in many species, even including 11 or 12 Drosophila species. Our data also showed that the functional bias observed in these young duplicate genes was mainly related to responses to environmental stimuli and biotic stresses.
\end{abstract}

Conclusions: This study reveals the evolutionary patterns of young duplicates across 12 Drosophila species on a genomic scale. Our results suggest that convergent evolution acts on young duplicate genes after the species differentiation and adaptive evolution may play an important role in duplicate genes for adaption to ecological factors and environmental changes in Drosophila.

Keywords: Young duplication, Environmental factor, Convergent evolution, Adaptive evolution

\section{Background}

Gene duplication is one of the dominant driving forces in adaptive evolution of genome and genetic systems [1]. Duplicate genes are considered to be the raw materials and the primary mechanism for generation of novel gene functions [2]. At least $15 \%$ of genes in human genome and $8 \%$ to $20 \%$ of Drosophila melanogaster, Caenorhabditis elegans, and Saccharomyces cervisiae genomes are thought to arise from gene duplications [3].

Young duplicate genes will ultimately suffer one of three long-term fates: (i) one copy may lose gene function by nonfunctionalization/pseudogenization; (ii) one copy may evolve a new beneficial function by means of neofunctionalization and the other retain the old; or (iii) both duplicated copies may stably be maintained with daughter copy partitioning of ancestral gene function by

\footnotetext{
* Correspondence: sihaiyang@nju.edu.cn; xiaohuizhang@nju.edu.cn ${ }^{\dagger}$ Equal contributors

State Key Laboratory of Pharmaceutical Biotechnology, School of Life Sciences, Nanjing University, 22 Hankou Rd., Nanjing 210093, China
}

subfunctionalization [3-7]. Many models have been proposed that pseudogenization could be the most common fate of duplicated genes [8-10]. In addition, evidences for adaptive evolution of pseudogenes have been reported in many organisms, such as pseudogenes in 80 Arabidopsis accessions [11] and the rcsA gene in Yersinia pestis [12]. Similarly, the preservation of duplicated genes might be a by-product of neutral evolution $[1,9,13]$, or might be adaptive substitutions during or after fixation of duplicates [1], indicating that selection for neofunctionalization is the mechanism to keep them $[14,15]$.

Previous studies conducted in many organisms have widely reported that duplicate genes undergo adaptive evolution. At the genome-wide level, the signatures of adaptive natural selection of young gene duplicates are found with high frequency in the human, macaque, mouse and rat genomes [16]. Furthermore, gene duplicates from Drosophila pseudoobscura neo-X chromosome [17] and a group of digestive protease encoding genes that are associated with recent, lineage-specific 
duplications in Drosophila Arizonae [18] are detected under adaptive evolution. Researches have been focused almost either on recent duplication events occurring in humans or other mammals involved in human diseases $[19,20]$ or on the duplication and adaptive evolution of single gene families, such as chalcone synthase genes and MADS-box genes in plants [21,22], fatty acid biosynthesis genes in bacteria [23], or Toll-like receptor genes in Drosophila [24]. Although gene gain and loss is estimated with a Drosophila-wide perspective [25], a systematic investigation of the genetic character and evolutionary pattern of young duplicate genes across the closely related Drosophila species has not been reported.

Sequencing of the genomes of the 12 worldwide Drosophila species (Drosophila 12 Genomes Consortium 2007) [26] provides the opportunity to reveal the evolutionary genetics of recent duplications. These species capture a range of evolutionary distances: closely related sister-species, such as $D$. simulans and D. sechellia or D. pseudoobscura and $D$. persimilis; distantly related species classified into different subgenera, such as Sophophora and Drosophila. There are also cosmopolitan species such as $D$. melanogaster and $D$. simulans or highly restricted species such as $D$. sechellia, distributed in some specific geographic ranges [27]. Additionally, the diverse host preferences provide a way to connect recent duplications with ecological and environmental factors. In this work, we conducted a genome-wide investigation of young duplicate genes across 12 Drosophila species to uncover their evolutionary patterns.

\section{Results}

Young duplicate genes in 12 Drosophila genomes

Across the 12 Drosophila genomes, a total of 22,488 gene families were detected, including 3,647 young duplicate gene families (see Methods; Table 1), suggesting that approximately $16.2 \%$ of the total gene families included young duplicates. In these young duplicate gene families, three types were defined based on their expansion patterns: species-specific expansions, lineagespecific expansions and complex expansions. The species-specific young duplicate gene families clearly predominated $(3159 / 3647=86.6 \%)$ over the other two types of expansions. On the other hand, uneven distribution of the species-specific young duplicate genes among different species, ranging from 54 to 794, was also observed. For example, $D$. melanogaster had the least family number (54), while the highest three values were found in D. willistoni (318), D. yakuba (569) and D. grimshawi (794), respectively (Table 1 ). This uneven distribution of the young duplicate genes was also found in the lineage-specific expansions and the complex expansions. For example, 114 duplicate gene families were detected in lineages of $D$. persimilis and D. pseudobsura, which is approximately 11.9 - and 4.9-fold greater than that in lineages of $D$. erecta and $D$. yakuba or $D$. sechellia and $D$. simulans, respectively. Also as expected, if there are more species (e.g. > 3) in a group of lineagespecific and complex expansions, fewer duplicate gene families were detected.

Table 1 Number of young duplicate gene families for three types of expansion

\begin{tabular}{|c|c|c|c|c|c|c|c|c|c|c|c|c|c|c|c|c|c|c|c|c|c|c|c|c|c|c|c|c|}
\hline \multirow{2}{*}{ Species } & \multirow{2}{*}{$\begin{array}{l}\text { Species- } \\
\text { specific } \\
\text { expansions }\end{array}$} & \multicolumn{6}{|c|}{ Lineage-specific expansions } & \multicolumn{20}{|c|}{ Complex expansions } & \multirow[t]{2}{*}{ Total } \\
\hline & & 2 & 2 & 2 & 3 & 5 & 6 & & 2 & & 3 & 4 & & 5 & & 6 & 6 & 7 & 8 & 8 & & 9 & 10 & 1 & & 11 & 12 & \\
\hline D. simulans & 139 & $+^{a}$ & - & - & + & + & + & - & & - & & + & & + & & - & + & + & + & & + & & + & & + & + & + & \\
\hline D. sechellia & 289 & + & - & - & + & + & + & - & & - & & + & & + & & - & - & - & + & & + & & - & & + & + & + & \\
\hline D. melanogaster & 54 & $-b$ & - & - & + & + & + & - & & - & & + & & + & & + & + & + & + & & + & & + & & + & + & + & \\
\hline D. yakuba & 569 & - & + & - & - & + & + & + & & - & & + & & - & & + & + & + & + & & + & & + & & + & + & + & \\
\hline D. erecta & 89 & - & + & - & - & + & + & - & & - & & - & & + & & + & + & + & + & & + & & + & & + & + & + & \\
\hline D. ananassae & 150 & - & - & - & - & - & + & - & c & - & & - & & + & & - & - & - & + & & + & $\cdots$ & + & & + & + & + & \\
\hline D. pseudobsura & 247 & - & - & + & - & - & - & - & & - & & - & & - & $\cdots$ & - & + & + & + & $\cdots$ & + & & + & $\cdots$ & + & - & + & \\
\hline D. persimilis & 232 & - & - & + & - & - & - & - & & - & & - & & - & & - & + & + & + & & + & & + & & + & + & + & \\
\hline D. willistoni & 318 & - & - & - & - & - & - & - & & - & & - & & - & & + & - & - & - & & + & & + & & + & + & + & \\
\hline D. virilis & 118 & - & - & - & - & - & - & - & & + & & - & & - & & + & - & + & - & & - & & + & & + & + & + & \\
\hline D. mojavensis & 160 & - & - & - & - & - & - & - & & + & & - & & - & & - & - & - & - & & - & & + & & - & + & + & \\
\hline \multirow[t]{2}{*}{ D. grimshawi } & 794 & - & - & - & - & - & - & + & & + & & - & & - & & + & - & - & - & & - & & - & & + & + & + & \\
\hline & & 29 & 12 & 143 & 11 & 5 & 1 & 21 & 177 & 5 & 44 & 3 & 9 & 1 & 6 & 1 & 1 & 1 & 1 & 3 & 2 & 3 & 1 & 2 & 1 & 2 & 3 & \\
\hline Total & 3159 & & & 201 & & & & & & & & & & & & & 2 & 87 & & & & & & & & & & 3647 \\
\hline
\end{tabular}

${ }^{a}$ Corresponding species is involved in the relevant expansion events.

${ }^{b}$ Corresponding species is not involved in the relevant expansion events.

${ }^{c}$ Not all expansion events are showed because of space limitation. 
Interestingly, three young duplicate gene families were detected respectively in complex expansions occurring in 11 and 12 species (Table 1). Although the six families were classified as the complex expansion type, speciesspecific duplication events also were found in all of these families (Additional file 1: Figure S1), especially independent duplications after the species differentiation in many species. For example, 15, 16 and 91 speciesspecific duplicate clades across all of the 12 species were detected in family $2,419,7,827$ and 8,177 , respectively. In the other 3 families, 8,11 and 145 species-specific duplicate clades were distributed in 8, 11 and 11 species, respectively. In addition, some lineage-specific duplications were also found in these families. All these results suggested that these duplicate gene families were likely to have been shaped by convergent evolution due to independent expansions in many species after the species differentiation.

\section{Distribution of young duplicate genes on chromosomes}

To explore the distribution of the young duplicate genes on the chromosomes, stochastic simulations were implemented using the observed gene numbers with 10,000 times random repeats. The chromosomal distribution was significantly non-random $(P<0.05)$; for example, chromosome 2 ( $2 \mathrm{~L} \& 2 \mathrm{R}), 3$ ( $3 \mathrm{~L} \& 3 \mathrm{R}$ ) and $\mathrm{X}$ contained a mass of young duplicate genes (Additional file 2: Figure S2). Figure 1 shows graphs representing these simulation results. Furthermore, the windows in Figure 1 with the observed number larger than the upper level of the confidence intervals correspond to hotspot regions on the chromosomes for the young duplications.

As shown in Figure 1, hotspot regions were found in all three types of young duplications, especially in speciesspecific expansions (Figure 1A), e.g. on chromosome 2 in D. grimshawi and on chromosome 3 in D. yakuba. In contrast, few hotspot regions were found in species such as in $D$. ananassae and D. melanogaster. Interestingly, some duplication hotspot regions were shared by more than one species in the species-specific expansions (marked by dash lines in Figure 1A), also suggesting convergent evolution of these genes among different species. However, none of shared hotspot region were detected in lineage-specific duplications, although the two species had similar trend lines which were generated by the observations and simulation numbers (Figure 1B). In complex expansions, few hotspot regions were detected along the chromosomes (Figure 1C).

\section{Functional preference of young duplicate genes}

To further reveal the genetic characteristics of the young duplications, the domains of the duplicates were detected using Pfam searches. Subsequently, the protein domains were counted in each species. For species- specific expansions, a total of 1,277 different domains were found in 12 species, averaging 106 protein domains in each species (Additional file 3: Table S1). Interestingly, approximately $84 \%$ of protein domains occurred only once or twice, suggesting that most domains were unique. However, the frequency of some protein domains, such as DUF1676 in D. willistoni, annexin and dynein_IC2 in D. melanogaster, inositol_P and PAP2 in D. yakuba, were high in one species but low ( 0 or 1 ) across the other 11 species, suggesting that these species-specific duplicate proteins might be driven by adaptive evolution in each species. Furthermore, some protein domains occurred in a lineage-specific manner, although they were detected in the species-specific expansion events. For example, the expansion of domains Gb3_synth and Gly_transf_sug shared by D. mojavensis and $D$. virilis, were greatly expanded only in these two species. A similar situation was also observed in the alpha-amylase domain, which occurred in two closely related Drosophila species, D. sechellia and D. simulans. Although different types and numbers of protein domains were examined in each species, we still found that approximately $4 \%$ of the domains appeared simultaneously in $\geq 6$ species. Prominent examples of these protein domains were trypsin, p450 and WD40, which were detected in 12, 11 and 11 species, respectively (Additional file 3: Table S1). These proteins are all important in response to environmental stimuli $[28,29]$. To investigate whether the high-frequency domains also occupied in large numbers in each species or vice versa, we examined the occurrencefrequency of the top 20 domains in 12 genomes. Interestingly, these high-frequency domains also had a large number of copies in the related species (Figure 2A), suggesting that these high-frequency duplicated proteins play an important role in the evolution of these species.

An identical approach was also used for the gene families of lineage-specific expansions and complex expansions. Our results showed that most gene families contained limited protein domains, although the number of the same domain was always different. However, some protein domains were still undergoing rapid expansion independently in many species, e.g. the six shared duplicate gene families in complex expansions occurring in 11 and 12 species (Table 1; Figure 2B and C). Furthermore, Ank, EGF, Peptidase_M17 and Peptidase_M17_N, which were all conserved and widespread domains in organisms for survival, exhibited high frequencies in 11 species (Figure 2B). In the shared expansion events of 12 species (Figure 2C), 12 of the top 20 protein domains such as histones, HSP70, Lys, co-occurred in 12 species. Numerous previous studies have shown that these protein domains are closely related to stress responses and pathogens in the environment, for example, histones are involved in stress responses [30], HSP70 protects cells 
Thong et al. BMC Genomics 2013, 14:555

Page 4 of 12

http://www.biomedcentral.com/1471-2164/14/555
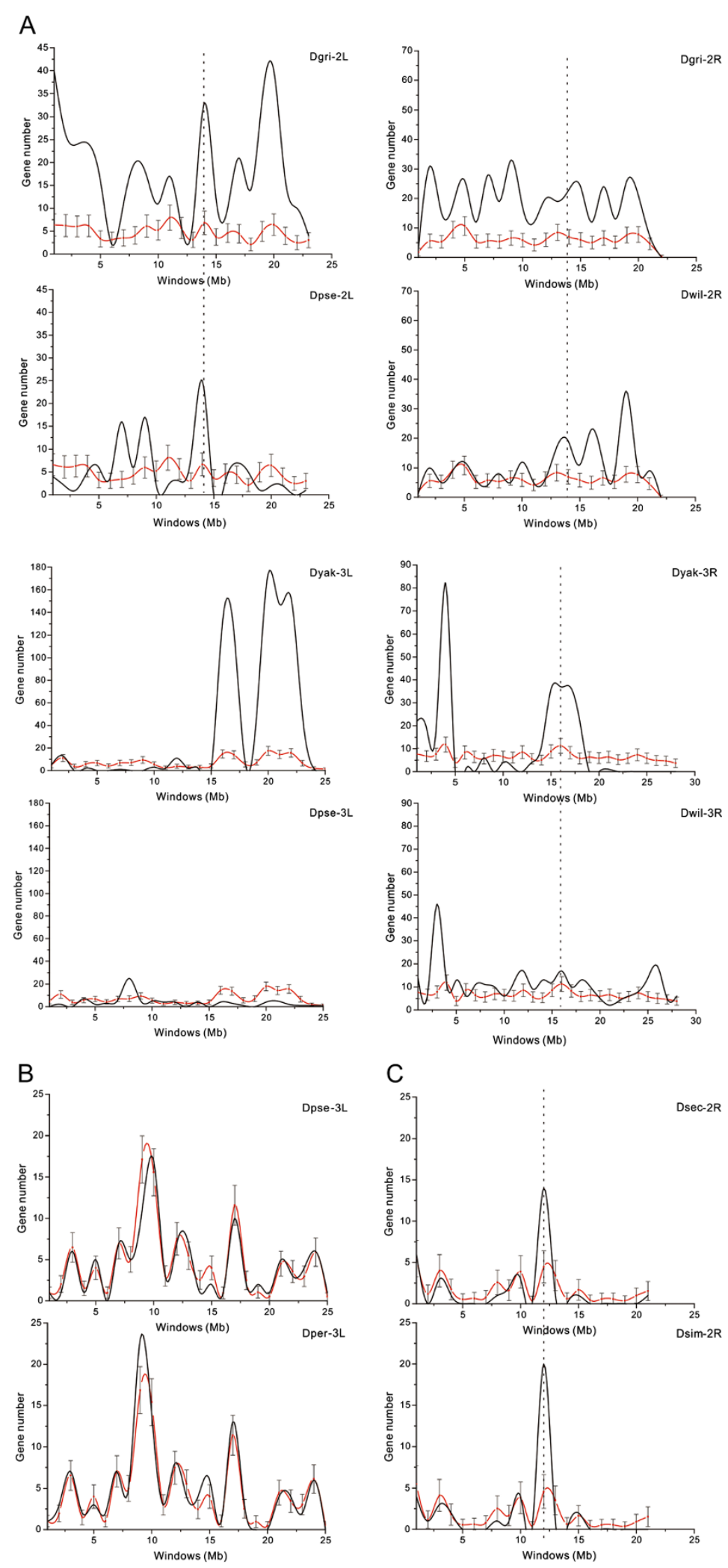

Figure 1 (See legend on next page.) 
(See figure on previous page.)

Figure 1 Distribution of young duplicate genes on chromosomes. (A) Species-specific duplicates on chromosome 2 and 3. (B) Lineage-specific duplicates of D. pseudoobscura-D. persimilis pair on chromosome 3. (C) Duplicates of complex expansions occurring in 11 species on chromosome 2. Black lines represent observations, while black bands and red lines (red dots) show confidence intervals and average numbers of genes in corresponding windows. Dashed lines indicate the shared hotspot regions between the species. Dgri: D. grimshawi, Dpse: D. pseudoobscura, Dper: D. persimilis, Dsec: D. sechellia, Dsim: D. simulans, Dwil: D. willistoni, Dyak: D. yakuba; 2L: chromosome 2L, 2R: chromosome 2R, 3L: chromosome 3L, 3R: chromosome $3 R$.

from stress [31], and Lys (lysozyme) acts as a bacteriolytic enzyme by hydrolyzing bacterial cell walls [32], suggesting that these shared duplications play an important role in adaption to ecological factors and environmental changes in Drosophila.

\section{Nonsynonymous and synonymous substitution between paralogs and orthologs}

The ratio of nonsynonymous to synonymous nucleotide substitution $(K a / K s)$ is considered as an important parameter indicating the strength of functional constraints. The smaller the $K a / K s$ ratio is, the stronger the functional constraints are. The 12 Drosophila whole-genome data offer us unprecedented opportunity to explore the different selection pressure between paralogs and orthologs. Therefore, we examined $K a / K s$ ratios for paralogs and orthologs in each duplicate gene family.

The average $K a / K s$ between paralog gene pairs or ortholog gene pairs in these young duplicate gene families were 0.626 and 0.445 , respectively, which was significantly $(P<0.05)$ larger than the genome-wide $\mathrm{Ka} / \mathrm{Ks}$ $(0.218)$ between ortholog pairs, suggesting relaxation of the functional constraints in the young duplicate gene families. Figure 3 shows that most of the gene pairs (91.2\%), including paralogs and orthologs, had $\mathrm{Ka} / \mathrm{Ks}$ ratios less than 1, demonstrating that most young duplicate genes were under purifying selection. However, there were still 229 and 82 gene pairs with $\mathrm{Ka} / \mathrm{Ks}$ ratios greater than 1 for paralogs and orthologs, respectively, indicating that some young duplicate genes are driven by positive selection. However, in the gene pairs with $K a / K s$ values exceeding 1 , many values were just slightly greater than 1 and only few pairs were detected to have $K a / K s$ ratios significantly greater than 1 .

Based on the strengths of boxes and whisker lines in species-specific expansions in Figure 3, it was clear that $K a / K s$ between paralogs had a broader dispersed distribution, larger median and quartile values than orthologs, indicating that paralogs had higher $\mathrm{Ka} / \mathrm{Ks}$ than orthologs. Similar results were also obtained in lineage-specific expansions (Figure 3D), with the exception of $D$. sechellia vs. $D$. simulans and D. persimilis vs. D. pseudoobscura. To further ensure that the $K a / K s$ of paralogs were significantly greater than those of orthologs, we conducted paired $t$-tests. Apart from four pairs, the other $\mathrm{Ka} / \mathrm{Ks}$ ratios of paralogs and orthologs exhibited highly significant $(P<0.01)$ or significant $(P<0.05)$ differences (Additional file 4: Table S2). All the results showed that paralogs had significantly higher $\mathrm{Ka} / \mathrm{Ks}$ than orthologs and indicated that paralogs are subject to weaker functional constraints and faster evolutionary processes than orthologs.

Linear analog was also performed between the mean $\mathrm{Ka} /$ $K s$ of paralogs and orhtologs (Additional file 5: Figure S3). In the same family, the dot above the trend lines (slope $=1$ ) indicated that paralogs have higher evolutionary rates than orthologs. Interestingly, it was clear that some dots lay far away the trend lines. Detection of the protein domains of these dots (Additional file 4: Table S2) showed that most of the domains detected in the genes of upper dots, such as Coesterase [33,34], Turandot [35] and MIP [36] were
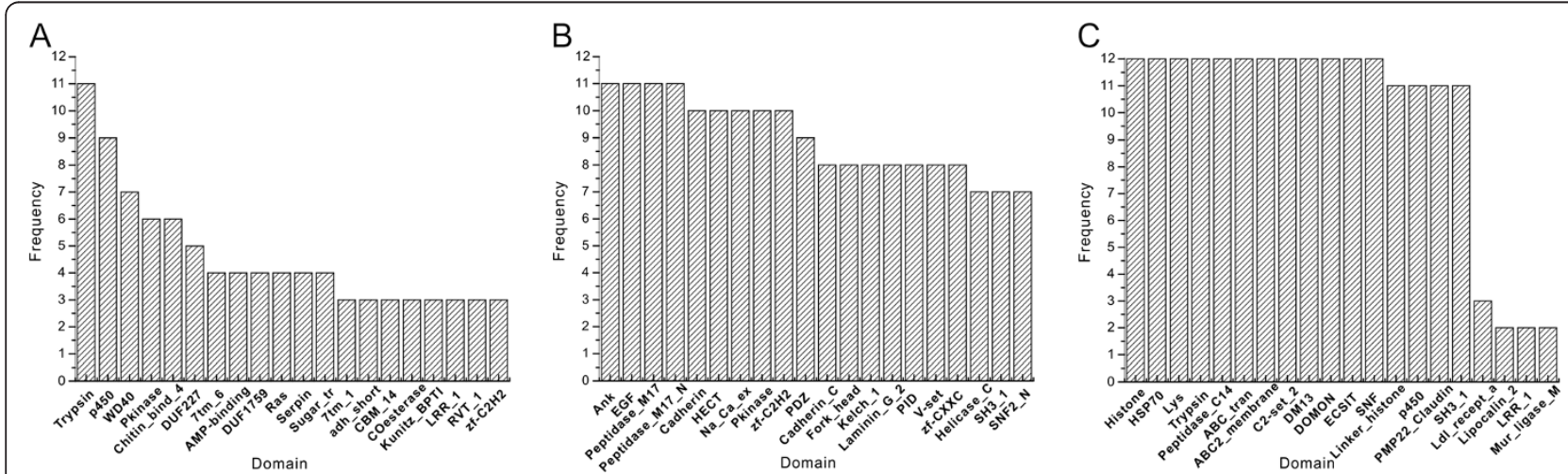

Figure $\mathbf{2}$ Occurrence-frequency of the top $\mathbf{2 0}$ domains in two types of expansions. (A) The frequency for species-specific expansions. (B) and $(\mathbf{C})$ The frequency for complex expansions occurring in 11 and 12 species, respectively. 

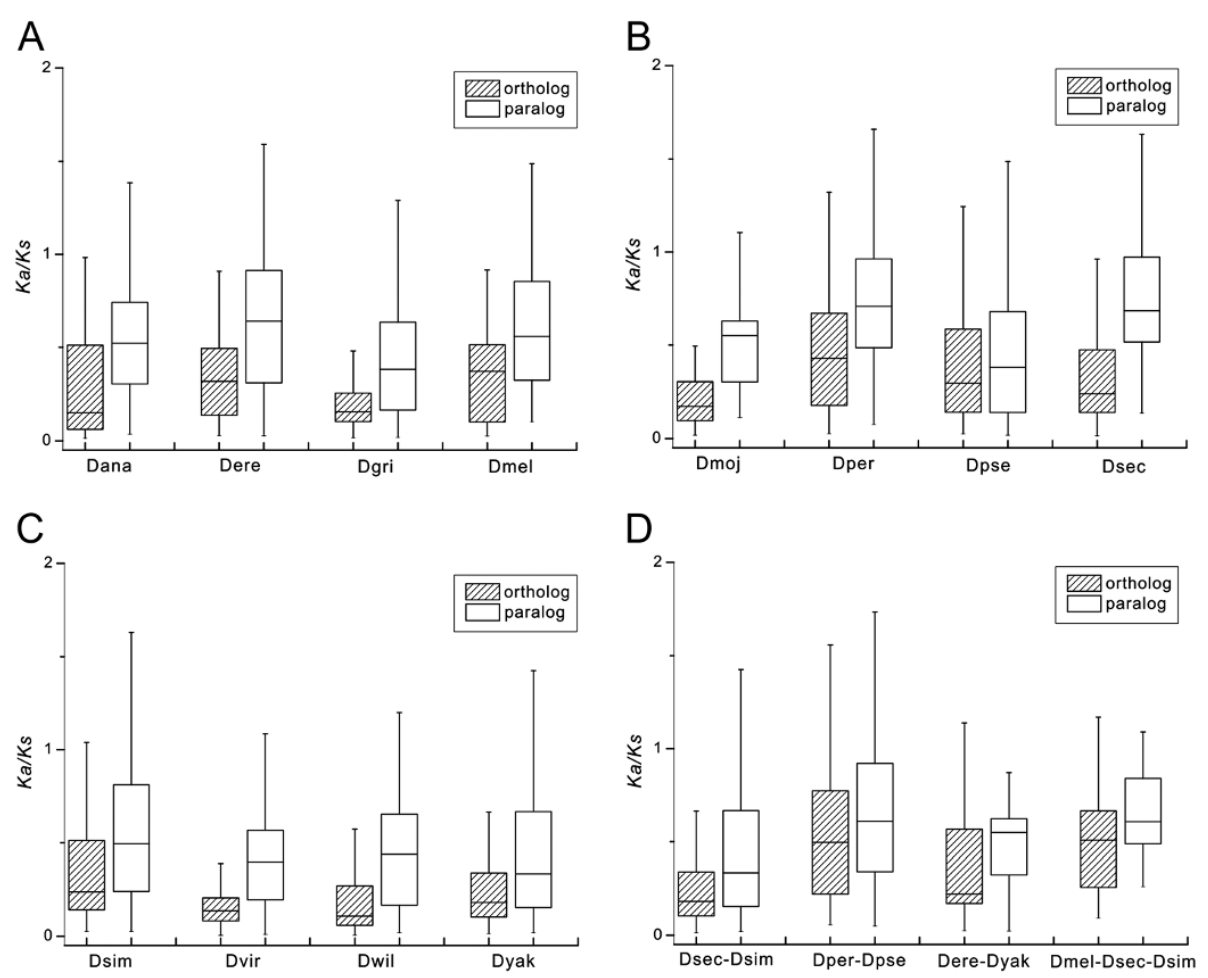

Figure 3 Box plot comparing the mean $K a / K s$ ratios of paralog gene pairs and ortholog gene pairs. (A), (B) and (C) $K a / K s$ ratios of species-specific duplicates. (D) Ka/Ks ratios of lineage-specific duplicates. The top bar is maximum observation, the lower bar is minimum observation, the top of box is third quartile, the bottom of box is lower or first quartile, the middle bar in box is median value.

involved in stress responses. These results also suggested that the young duplicates result from adaption to the environment both in species-specific and lineage-specific expansions.

\section{Evolutionary analysis of young duplicate genes across 12 Drosophila species}

To detect the timing of recent duplication in each species, the $K s$ values were calculated. We adopted the common assumption that Drosophila species experienced about 10 generations/year and that the single nucleotide mutation rate was $5.8 \times 10^{-9}$ mutations per generation [37]. Furthermore, only $K s$ values lower than 1.0 were kept to avoid the saturation of nucleotide substitutions.

On the whole, the young duplication events occurred over a short span of time (0.082-5.282 MYA). The duplication time of species-specific expansions fell in a range from 1.238 MYA (D. simulans) to 3.573 MYA (D. melanogaster) (standard deviation, 0.712) (Table 2), indicating that all the species-specific expansions occurred within a short time. Moreover, most of our estimates of duplication time were less than the species divergence time reported by Tamura [38]. However, the divergence time of several closely related species previously reported, including D. simulans vs. D. sechellia $(<0.93 \pm 0.49$ MYA) and D. pseudoobscura vs. D. persimilis $(0.85 \pm 0.29$ MYA $)$ was slightly lower than their respective family duplication times (1.238, 2.313 and 1.327, 1.573 MYA). Similarly, higher duplication times in the four species were also found in lineage-specific expansions and complex expansions. Moreover, the lowest standard deviations of the duplication time were detected between these lineage species in lineage-specific expansions, which suggested that closely related species duplicated in close periods, especially the species pair $D$. persimilis vs. D. pseudoobscura (2.341 and 2.401 MYA). In the six lineage species, there was a group of duplication times with more compact distribution and smaller values than those in species-specific expansions, which indicated that the expansion occurred over a more concentrated and closer period in lineagespecific expansions than in species-specific expansions. It was clear that less species and closer relationships caused such results. Finally, although the highest standard deviations were found in complex expansions, especially in 11 species with a broader range for duplication time (0.7655.282 MYA) and a larger standard deviation (1.686) than those in others, their distributions of duplication time were still in relative compact period. This demonstrated that these duplicated genes in complex expansions might appear at relatively scattered time compared with 
Table 2 Duplication time for species-specific expansions, lineage-specific expansions and complex expansions

\begin{tabular}{|c|c|c|c|c|c|c|c|c|c|c|}
\hline \multirow{2}{*}{$\frac{\text { Species }}{\text { D. simulans }}$} & \multirow{2}{*}{$\begin{array}{c}\begin{array}{c}\text { Species-specific } \\
\text { expansions }\end{array} \\
1.238\end{array}$} & \multicolumn{6}{|c|}{ Lineage-specific expansions } & \multicolumn{3}{|c|}{ Complex expansions } \\
\hline & & 1.561 & - & - & 1.492 & 1.916 & 0.981 & 2.763 & 1.531 & 1.655 \\
\hline D. sechellia & 2.313 & 2.249 & - & - & 1.281 & 1.575 & 0.084 & 2.576 & 0.765 & 0.525 \\
\hline D. melanogaster & 3.573 & - & - & - & 1.022 & 0.769 & 0.998 & 0.674 & 1.169 & 3.794 \\
\hline D. yakuba & 1.613 & - & 3.526 & - & - & 0.641 & 0.564 & 4.843 & 1.644 & 2.549 \\
\hline D. erecta & 1.874 & - & 2.775 & - & - & 0.539 & 0.082 & 5.131 & 1.287 & 0.760 \\
\hline D. ananassae & 2.572 & - & - & - & - & - & 0.582 & 3.372 & 5.282 & 1.244 \\
\hline D. pseudoobscura & 1.327 & - & - & 2.401 & - & - & - & 4.108 & - & 3.790 \\
\hline D. persimilis & 1.573 & - & - & 2.341 & - & - & - & 3.268 & 1.017 & 4.490 \\
\hline D. willistoni & 2.085 & - & - & - & - & - & - & 2.419 & 2.560 & 1.473 \\
\hline D. virilis & 2.513 & - & - & - & - & - & - & 3.509 & 5.153 & 1.003 \\
\hline D. mojavensis & 3.388 & - & - & - & - & - & - & - & 1.332 & 1.023 \\
\hline D. grimshawi & 2.160 & - & - & - & - & - & - & 2.162 & 4.755 & 0.449 \\
\hline Standard deviation & 0.712 & 0.344 & 0.375 & 0.030 & 0.192 & 0.553 & 0.371 & 1.207 & 1.686 & 1.349 \\
\hline
\end{tabular}

duplicates in the other two types. Therefore, we might infer that Drosophila species have consistently duplicated to adapt to environmental changes.

\section{Discussion}

\section{Convergent evolution of young duplicate genes across} the 12 Drosophila species

Convergent evolution plays an important role in biological adaptation, by which distantly related organisms independently evolve similar structures or functions in order to adapt to similar environments or ecological niches [39], such as, the specialized oxygen transport function of oxygen transport hemoglobins in jawed and jawless vertebrates [40] and the similar substrate of apolipoprotein (a) in humans and hedgehogs [41]. Although there are many other theories could explain the evolutionary process of young duplicates, such as genomic drift proposed by Nei [42,43], convergent evolution might be more convincible for two evidences detected in our study.

In our study, the phylogenetic trees (Additional file 1: Figure S1) and the chromosomal distributions (Figure 1) of young duplicate genes also provide evidence of convergent evolution. Six young duplicate families were found in complex expansions occurring in 11 or 12 species with many species-specific duplication clades across these 11 or 12 species. Interestingly, the phenomenon that the independent duplicates with similar function preference are under convergent evolution has also been previously reported both in animals and plants. For example, histone proteins are highly alkaline proteins in eukaryotic genomes which package DNA into nucleosomes [44] and independent convergent evolution has produced striking similarities between plant and animal histones [45]. Another example of similar genetic charac- teristics shared by distant species is the digestion function of lysozymes (Lys domain) in animals. Lysozymes are usually present in tears, saliva and other bodily fluids and have independently been recruited to the stomach and play important roles in enzyme functions across vertebrates [46]. Furthermore, some duplication hotspot regions were shared by more than one species across their chromosomes in species-specific expansions. Interestingly, conserved duplication hotspots have also been previously detected between D. melanogaster and D. simulans [47]. Similar function preference and identical hotspot regions arising from independent duplications suggest that the young duplicate genes have undergone convergent evolution which appears to have played an important role in the independent evolution of adaptive traits in 12 Drosophila species.

\section{Adaptive evolution supported by functional bias of young duplicates}

It is well-known that duplicate genes face three possible fates: pseudogenization, subfunctionalization and neofunctionalization. Pseudogenization is considered as the most common fate of duplicate genes [8-10], but more evidence support the models of subfunctionalization or neofunctionalization, as the mechanisms for the preservation of duplicate genes under adaptive selection $[6,15,48,49]$. Many previous studies have shown that the duplicated genes could adapt to various conditions, in particular, genes encoding membrane or secreted proteins which are always involved in ecological stimuli or stress. For example, adaptive gene duplications have been found in response to biotic stress [50], antibiotics [51,52], weedicides [53] or pesticides [54,55], drugs or toxins [56], extreme temperatures $[57,58]$, nutrient limitation $[59,60]$ and symbiosis between host and parasite [61]. 
In this study, it was shown that the protein domains of trypsin, p450, WD40 and Pkinase in species-specific expansions, Ank, EGF, histone, HSP70 and Lys in complex expansions occurred with high frequency across the 12 Drosophila species. Interestingly, these young duplicates were also involved in different aspects of interactions with the environment. Trypsin is one of the largest families of secreted serine proteases found in the digestive system of vertebrates and invertebrates. Although it participates in many basic physiological processes [62-64], it is predominantly involved in diet and digestion. The high frequency of trypsin across 12 Drosophila species indicated that consistent and independent duplication for adaptation to specific dietary habits was due to their diverse ecosystems [27,29]. For example, investigations of trypsin family conducted in various genomes, such as fruit fly [65], mosquitoes [66] and leaf-eating monkey [67], have all indicated that adaption occurs in response to specific diets. In particular, researches into the rapid diversification of trypsin genes in 12 Drosophila species have provided insights into the ecological forces driving the adaptive evolution by comparing the relationship between duplications and host preference shifts [65].

Another protein domain shared between 11 Drosophila species detected in this study was cytochrome p450 (CYP). P450 comprise a superfamily of enzymes that occurs with a high degree of diversity in all organisms [68]. Among the various biological functions of $\mathrm{p} 450$, we focused on the oxidation of xenobiotic compounds, which facilitates their excretion from the organism $[69,70]$. Abuse of insecticides has forced adaptive evolution in Drosophila over an extremely short period. A single p450 gene, Cyp6g1, is sufficient and necessary for DDT resistance [28] and its cross-resistance to a wide range of other insecticides has also been identified in Drosophila [71,72]. Furthermore, functional divergence and positive selection detected in mammalian CYP genes, provide insights into the adaptive selection of CYPs in response to high diversity of xenobiotics [73].

Other expanded domains were also identified with roles in adaption to various ecological factors, especially stress. For example, some SAPK (stress-active protein kinases, Pkinase) mediate cellular responses to toxins and physical stress [74] and TAK1 (transforming growth factor- $\beta$-activate kinase, Pkinase) is a key regulator in response to diverse stimuli in adaptive immunity [75], ankyrin proteins (Ank) play a role in stress responses and disease resistance both in animals [76] and plants [77], histones [30] and HSP70 proteins protect cells from stress [31], and Lys proteins act as bacteriolytic enzymes by hydrolyzing cell bacterial walls [32].

These observations indicate that shared young duplications reflect adaptive evolution of the Drosophila species to global ecological pressures.

\section{Adaptive evolution contributes to specific functional preference}

In this study, although most paralogs and orthologs of these young duplicate gene families had $\mathrm{Ka} / \mathrm{Ks}$ ratios lower than 1 , some $K a / K s$ ratios greater than 1 were also found both in species-specific and lineage-specific expansions (Figure 3), demonstrating that they were under adaptive selection. Furthermore, paralog gene pairs had higher $\mathrm{Ka} / \mathrm{Ks}$ ratios than ortholog gene pairs across 12 Drosophila species. It can be concluded that the paralogs have higher frequency of adaptive evolution than the orthologs [48]. Previous research has indicated that many genes families in Drosophila are driven by adaptive selection, such as, elastase/chymotrypsin, trypsin and astacin, which are all involved in digestive processes in D. arizonae [26], two immunity-related gene families, Toll-like receptors and lysozyme in D. melanogaster $[24,78]$, and metallothionein genes involved in metal tolerance $[79,80]$. Moreover, positive selection is a major force driving the evolution of male-specific recent duplicates on neo-X chromosome in D. pseudoobscura [17] and segmentally duplicated seminal fluid genes in $D$. melanogaster [81].

Functional analysis of those gene families in which paralogs had higher $\mathrm{Ka} / \mathrm{Ks}$ ratios than orthologs with ratios exceeding 1 (Figure 3 ) showed that adaptive evolution leads to species-specific and lineage-specific functional preference for each Drosophila (Additional file 3: Table S1). Examples include the PDZ-domain containing gene family in $D$. sechellia, stress-inducible humoral factor Turandot (Turandot domain) in D. yakuba and the Pam16 family (Pam16 domain) in the D. pseudoobscura and $D$. persimilis pair. Combining the functional preference with the host preference of corresponding Drosophila species [27], it seems reasonable to infer that Drosophila evolve for adaption to a given environment [82].

Interestingly, we found that $D$. sechellia only distribute in the Seychelle Islands in the Indian Ocean and prefer inhabiting Morinda citrifolia, the fruit of which contain nutrients such as alkaloids. Alkaloids are widely known that play an important role in inhibiting tumors by reducing microtubules disruption during mitosis [83]. Coincidently, PDZ proteins are involved in the interaction between syntrophin-associated serine/threonine kinase and microtubule-associated serine/threonine kinases and are recognized as tumor suppressors [84,85]. Therefore, we speculated that duplication of PDZ in D. sechellia is closely associated with adaption to this unique habitat.

The D. yakuba species in Africa exhibited propagation of Turandot proteins which are adaptively resistant to high temperature, dehydration and UV irradiation [86,87]. In contrast, the Pam16 proteins of D. pseudoobscura and D. persimilis play important regulatory roles in recruiting heat shock protein partners and responding to cold harde- 
ning [88-90], indicating superior adaptation of these species to their specific habitats situated in the regions of higher latitude in the Northern Hemisphere compared with other species.

Furthermore, other evidence of adaptive evolution in a single species or lineage species pairs was also detected in domain analysis (Additional file 3: Table S1). For example, annexin and dynein_IC2 in D. melanogaster, which are sperm-specific proteins (annexin $\mathrm{X}$ and cytoplasmic dynein intermediate chain) and absent in other species of the melanogaster species subgroup, support the hypothesis that male reproductive functions are driven by selective sweep and rapid molecular evolution [91]. Another example is alpha-amylase (Amy), a digestive enzyme of the two closely related species of the 12 Drosophila, $D$. sechellia and $D$. simulans, for which genetic variation of duplicated amylase genes has been reported revealing adaptive evolution in Drosophila [92].

Consequently, adaptive evolution of Drosophila species leads to young duplicate genes exhibiting specific function preference in response to ecological factors and environmental changes.

\section{Conclusions}

We identified 3,647 young duplicate gene families across 12 Drosophila species and detected three types of expansions in these gene families: species-specific, lineage-specific and complex expansions. We found that the species-specific young duplicate genes predominated $(86.6 \%)$ over the other two types. Furthermore, we observed that, in the same gene family, independent species-specific expansions occurred in many species, even including 11 or 12 Drosophila species, suggesting that these young duplicate genes were under convergent evolution after the Drosophila species differentiation. We also found that the functional preference of the young duplicate genes was mainly related to responses to environmental stimuli and biotic stresses, suggesting that adaptive evolution may play an important role in duplicate genes for adaption to ecological factors in Drosophila species. This work may help us to better understand the evolutionary patterns of young duplicate genes across 12 Drosophila species.

\section{Methods}

\section{Identification of young duplicate genes}

The 12 Drosophila genome sequences and annotations were downloaded from the Flybase Datebase [ftp://ftp.flybase.net/ genomes/] [93], and the exact version for each species is shown in Table 1 (Additional file 4). An all-versus-all Blastn search with E-value (1.0e-40) was processed across all nucleotide coding sequences (CDSs) in 12 Drosophila species, then coverage of $>60 \%$ was used to divide the genes into gene families. Subsequently, Clustalw2.0 [94] was used for the pairwise alignment of the nucleotide sequences of genes in one family and the nucleotide diversity $(\pi)$ was estimated with the Jukes and Cantor correction by MEGA v5.0 [95]. Young duplicate gene families were defined based on the following two conditions: (1) the number of family members was larger than the number of corresponding species in each family; (2) the highest identity of the paralogs in each family exceeded $90 \%$.

To further analyze young duplicate gene families, three types of expansions were defined: species-specific expansions, lineage-specific expansions and complex expansions. Here, the species-specific expansions were denoted as young duplications occurring only in one species, while other species comprised $\leq 1$ member or $>2$ members but with less than $90 \%$ identity between paralogs. We also defined the latter two types as the young duplications of a gene family in species with a close (lineage-specific expansion) or distant (complex expansion) genetic relationship based on the species tree of the 12 Drosophila species [27]. Based on this principle, species-specific expansions were easily identified corresponding to each species. Furthermore, we defined the following six lineage-specific expansions: $D$. sechellia-D. simulans, $D$. yakuba-D. erecta, $D$. pseudoobscura-D. persimilis, D. melanogaster-D. sechellia$D$. simulans, $D$. melanogaster- $D$. sechellia- $D$. simulans- $D$. yakuba-D. erecta and $D$. melanogaster-D. sechellia-D. simulans-D. yakuba-D. erecta-D. ananassae, and $14 \mathrm{com}-$ plex expansions (Table 1 \& Additional file 6: Table S4).

\section{Sequence alignment and phylogenetic analysis}

The amino acid sequences of the duplicate genes in each family were first aligned using the MUSCLE program with default options and then manually corrected using MEGA v5.0 [95]. The alignments were used to construct phylogenetic trees with 1,000 replicates using MEGA v5.0 based on the neighbor-joining (NJ) method [96]. NJ analysis was conducted using pairwise deletion of gaps and kimura-2 model (family3093, family9588 and family7827) or p-distance (family8177). Additionally, for the two families (family21 and family2419) with numerous members, $\mathrm{NJ}$ trees were constructed with default parameters and 1,000 bootstrap replicates in Clustalw2.0 [94].

\section{Physical location and structural domains of the young duplicate genes}

The hotspot regions for duplication events were examined by identifying the exact physical positions of young duplicate genes across the chromosomes. Detailed genome annotation information was available for the genes of D. melanogaster only. Thus, we performed Blast analysis using the CDSs of young duplicate genes in the other 11 genomes against the genome sequences of $D$. melanogaster to gain the position information.

We utilized the position information to process stochastic simulations with 10,000 random repeats by PERL 
script. Each chromosome was divided into a number of windows $(1 \mathrm{Mb} / 1$ Window). We incorporated all genes of corresponding species into the given window of each chromosome for the species-specific duplicate gene families, and an identical approach was taken for the families of lineage-specific expansions and complex expansions. All young duplicate genes identified in this study were further examined for structural domains using the Pfam database [Pfam database 26.0, http://pfam.sanger.ac.uk/] [97] with E-value 1.0.

\section{Calculation of nonsynonymous to synonymous ratio and estimation of duplication time of paralogs}

CDSs in each young duplicate gene family were aligned according to alignments of protein sequences in Clustalw2.0 [94]. Subsequently, the nonsynonymous substitutions $(\mathrm{Ka})$, synonymous substitutions $(K s)$ and ratio of nonsynonymous to synonymous substitutions $(K a / K s)$ were calculated for paralog and ortholog pairs in each duplicate gene family using MEGA v5.0 [95].

The mean $K s$ values were calculated for paralog pairs in each species-specific duplicated gene family and then used to estimate the timing of duplications. The calculations were performed using a single nucleotide mutation rate of $5.8 \times 10^{-9}$ mutations per generation and it was assumed that Drosophila species experienced approximately 10 generations/year [37].

\section{Additional files}

Additional file 1: Figure S1. Phylogenetic trees of the six gene families of complex expansions occurring in 11 and 12 species. Dana: $D$. ananassae, Dere: D. erecta, Dgri: D. grimshawi, Dmoj: D. mojavensis, Dper: D. persimilis, Dpse: D. pseudoobscura, Dsec: D. sechellia, Dsim: D. simulans, Dvir: D. virilis, Dwil: D. willistoni, Dyak: D. yakuba. Genes without abbreviative species name are from $D$. melanogaster.

Additional file 2: Figure S2. Distribution of young duplicate genes on chromosomes. (A)-(E) Species-specific duplicates on chromosome 2L, 2R, $3 L, 3 R$ and $X$, respectively. (F) Lineage-specific duplicates of $D$. pseudoobscura-D. persimilis pair. (G)-(K) Duplicates of complex expansions occurring in 11 species on chromosome $2 \mathrm{~L}, 2 \mathrm{R}, 3 \mathrm{~L}, 3 \mathrm{R}$ and $\mathrm{X}$, respectively. Black lines represent observations, while black bands and red lines (red dots) show confidence intervals and average numbers of genes in corresponding windows. Dmel: D. melanogaster. $2 \mathrm{~L}$ : chromosome 2L, 2R: chromosome 2R, 3L: chromosome 3L, 3R: chromosome 3R.

Additional file 3: Table S1. Number of protein domains for young duplicate genes in species-specific expansions.

Additional file 4: Table S2. Structural domains detected in families corresponding to the dots excessively deviating from trend lines. Table S3. Versions of genome sequences and annotations for each species.

Additional file 5: Figure S3. Mean Ka/Ks ratios of paralog gene pairs vs. ortholog gene pairs. (A) Ka/Ks ratios of species-specific duplicates. (B) $\mathrm{Ka} / \mathrm{Ks}$ ratios of lineage-specific duplicates. Black lines means the trend line of black dots and red lines represents trend lines with slope $=1$.

Additional file 6: Table S4. Number of young duplicate gene families for three types of expansion.

\section{Abbreviations}

CDS: Nucleotide coding sequences; MYA: Million years ago.
Competing interests

The authors declare that they have no competing interests.

\section{Authors' contributions}

$D T, S Y$ and $Y Z$ designed the study. YZ and YJ contributed extensively to the bioinformatic analyses. $Y Z, X Z$ and $Y G$ wrote the manuscript. $S Y, X Z$ and $Y Z$ prepared and revised the manuscript. All authors read and approved the final manuscript.

\section{Acknowledgements}

This work was supported by the National Natural Science Foundation of China (30970198, J1103512 and J1210026), Program for New Century Excellent Talents in University (NCET-12-0259), Changjiang Scholars and Innovative Research Team in University (IRT1020) and RFDP

(20090091110031).

Received: 29 December 2012 Accepted: 13 August 2013

Published: 15 August 2013

\section{References}

1. Ohno S: Evolution by gene duplication. London: George Alien \& Unwin Ltd. Berlin, Heidelberg and New York: Springer-Verlag; 1970.

2. Long M, Langley $\mathrm{CH}$ : Natural selection and the origin of jingwei, a chimeric processed functional gene in Drosophila. Science 1993, 260(5104):91-95.

3. Lynch M, Conery JS: The evolutionary fate and consequences of duplicate genes. Science 2000, 290(5494):1151-1155.

4. Lynch M, Force A: The probability of duplicate gene preservation by subfunctionalization. Genetics 2000, 154(1):459-473.

5. Lynch $\mathrm{M}$, O'Hely $\mathrm{M}$, Walsh $B$, Force $A$ : The probability of preservation of a newly arisen gene duplicate. Genetics 2001, 159(4):1789-1804.

6. Walsh JB: How often do duplicated genes evolve new functions? Genetics 1995, 139(1):421-428.

7. Wagner A: The fate of duplicated genes: loss or new function? Bioessays 1998, 20(10):785-788.

8. Hughes AL: The evolution of functionally novel proteins after gene duplication. Proc Biol Sci 1994, 256(1346):119-124.

9. Force A, Lynch M, Pickett FB, Amores A, Yan YL, Postlethwait J: Preservation of duplicate genes by complementary, degenerative mutations. Genetics 1999, 151(4):1531-1545.

10. Stoltzfus A: On the possibility of constructive neutral evolution. J Mol Evol 1999, 49(2):169-181.

11. Wang L, Si W, Yao Y, Tian D, Araki H, Yang S: Genome-Wide Survey of Pseudogenes in 80 Fully Re-sequenced <italic>Arabidopsis thaliana</italic> Accessions. PLOS ONE 2012, 7(12):e51769.

12. Sun $Y C$, Hinnebusch BJ, Darby C: Experimental evidence for negative selection in the evolution of a Yersinia pestis pseudogene. Proc Natl Acad Sci 2008, 105(23):8097-8101.

13. Dykhuizen D, Hartl DL: Selective neutrality of 6PGD allozymes in E. coli and the effects of genetic background. Genetics 1980, 96(4):801-817.

14. Rodriguez-Trelles F, Tarrio R, Ayala FJ: Convergent neofunctionalization by positive Darwinian selection after ancient recurrent duplications of the xanthine dehydrogenase gene. Proc Natl Acad Sci USA 2003, 100(23):13413-13417.

15. Thornton K, Long M: Excess of amino acid substitutions relative to polymorphism between X-linked duplications in Drosophila melanogaster. Mol Biol Evol 2005, 22(2):273-284.

16. Kuwabara $P E$, Labouesse $M$ : The sterol-sensing domain: multiple families, a unique role? Trends Genet 2002, 18(4):193-201.

17. Meisel RP, Hilldorfer BB, Koch JL, Lockton S, Schaeffer SW: Adaptive evolution of genes duplicated from the Drosophila pseudoobscura neoX chromosome. Mol Biol Evol 2010, 27(8):1963-1978.

18. Kelleher ES, Swanson WJ, Markow TA: Gene duplication and adaptive evolution of digestive proteases in Drosophila arizonae female reproductive tracts. PLoS Genet 2007, 3(8):e148.

19. Bailey JA, Gu Z, Clark RA, Reinert K, Samonte RV, Schwartz S, Adams MD, Myers EW, Li PW, Eichler EE: Recent segmental duplications in the human genome. Science 2002, 297(5583):1003-1007.

20. Cheng Z, Ventura M, She X, Khaitovich P, Graves T, Osoegawa K, Church D, DeJong P, Wilson RK, Paabo S, et al: A genome-wide comparison of recent 
chimpanzee and human segmental duplications. Nature 2005, 437(7055):88-93.

21. Yang J, Huang J, Gu H, Zhong Y, Yang Z: Duplication and adaptive evolution of the chalcone synthase genes of Dendranthema (Asteraceae). Mol Biol Evol 2002, 19(10):1752-1759.

22. Hernández-Hernández T, Martínez-Castilla LP, Alvarez-Buylla ER: Functional diversification of $B$ MADS-box homeotic regulators of flower development: adaptive evolution in protein-protein interaction domains after major gene duplication events. Mol Biol Evol 2007, 24(2):465-481.

23. Kinsella RJ, Fitzpatrick DA, Creevey CJ, Mclnerney JO: Fatty acid biosynthesis in Mycobacterium tuberculosis: lateral gene transfer, adaptive evolution, and gene duplication. Proc Natl Acad Sci 2003, 100(18):10320

24. Medzhitov R, Preston-Hurlburt P, Janeway CA Jr: A human homologue of the Drosophila Toll protein signals activation of adaptive immunity. Nature 1997, 388(6640):394-397.

25. Hahn MW, Han MV, Han SG: Gene family evolution across 12 Drosophila genomes. PLoS Genet 2007, 3(11):e197.

26. Stark A, Lin MF, Kheradpour P, Pedersen JS, Parts L, Carlson JW, Crosby MA Rasmussen MD, Roy S, Deoras AN: Discovery of functional elements in 12 Drosophila genomes using evolutionary signatures. Nature 2007, 450(7167):219-232

27. Singh ND, Larracuente AM, Sackton TB, Clark AG: Comparative genomics on the Drosophila phylogenetic tree. Annu Rev Ecol Evol Syst 2009, 40:459-480.

28. Daborn PJ, Yen JL, Bogwitz MR, Le Goff G, Feil E, Jeffers S, Tijet N, Perry T, Heckel D, Batterham P, et al: A single p450 allele associated with insecticide resistance in Drosophila. Science 2002, 297(5590):2253-2256.

29. Li L, Memon S, Fan Y, Yang S, Tan S: Recent duplications drive rapid diversification of trypsin genes in 12 Drosophila. Genetica 2012, 140(7-9):297-305.

30. Camporeale G, Zempleni J, Eissenberg JC: Susceptibility to heat stress and aberrant gene expression patterns in holocarboxylase synthetasedeficient Drosophila melanogaster are caused by decreased biotinylation of histones, not of carboxylases. J nutr 2007, 137(4):885-889.

31. Morano KA: New tricks for an old dog: the evolving world of Hsp70. Ann N Y Acad Sci 2007, 1113:1-14.

32. Powning R, Davidson W: Studies on insect bacteriolytic enzymes-II Some physical and enzymatic properties of lysozyme from haemolymph of Galleria mellonella. Comp Biochem Physiol B Comp Biochem 1976, 55(2):221-228.

33. Blackman R, Spence J, Field L, Javed N, Devine G, Devonshire A: Inheritance of the amplified esterase genes responsible for insecticide resistance in Myzus persicae (Homoptera: Aphididae). Heredity 1996, 77(2):154-167.

34. Hemingway J, Hawkes NJ, McCarroll L, Ranson H: The molecular basis of insecticide resistance in mosquitoes. Insect Biochem Mol Biol 2004, 34(7):653-665

35. Buchon N, Broderick NA, Poidevin M, Pradervand S, Lemaitre B: Drosophila intestinal response to bacterial infection: activation of host defense and stem cell proliferation. Cell Host Microbe 2009, 5(2):200-211.

36. Weig A, Deswarte C, Chrispeels MJ: The major intrinsic protein family of Arabidopsis has 23 members that form three distinct groups with functional aquaporins in each group. Plant Physiol 1997, 114(4):1347-1357.

37. Haag-Liautard C, Dorris M, Maside X, Macaskill S, Halligan DL, Houle D, Charlesworth B, Keightley PD: Direct estimation of per nucleotide and genomic deleterious mutation rates in Drosophila. Nature 2007, 445(7123):82-85.

38. Tamura K, Subramanian S, Kumar S: Temporal patterns of fruit fly (Drosophila) evolution revealed by mutation clocks. Mol Biol Evol 2004 21(1):36-44.

39. Doolittle RF: Convergent evolution: the need to be explicit. Trends Biochem Sci 1994, 19(1):15.

40. Hoffmann FG, Opazo JC, Storz JF: Gene cooption and convergent evolution of oxygen transport hemoglobins in jawed and jawless vertebrates. Proc Natl Acad Sci U S A 2010, 107(32):14274-14279.

41. Lawn RM, Schwartz K, Patthy L: Convergent evolution of apolipoprotein(a) in primates and hedgehog. Proc Natl Acad Sci U S A 1997, 94(22):11992-11997.

42. Nozawa M, Kawahara Y, Nei M: Genomic drift and copy number variation of sensory receptor genes in humans. Proc Natl Acad Sci 2007, 104(51):20421-20426.
43. Nozawa M, Nei M: Evolutionary dynamics of olfactory receptor genes in Drosophila species. Proc Natl Acad Sci 2007, 104(17):7122-7127.

44. Youngson RM: Collins Dictionary of Human Biology. Glasgow: HarperCollins 2006:352.

45. Fambrough DM, Bonner J: On the Similarity of Plant and Animal Histones*. Biochemistry 1966, 5(8):2563-2570.

46. Arendt J, Reznick D: Convergence and parallelism reconsidered: what have we learned about the genetics of adaptation? Trends Ecol Evol 2008, 23(1):26-32.

47. Cardoso-Moreira M, Emerson JJ, Clark AG, Long M: Drosophila duplication hotspots are associated with late-replicating regions of the genome. PLOS genetics 2011, 7(11):e1002340.

48. Han MV, Demuth JP, McGrath CL, Casola C, Hahn MW: Adaptive evolution of young gene duplicates in mammals. Genome Res 2009, 19(5):859-867.

49. Kondrashov FA, Rogozin IB, Wolf YI, Koonin EV: Selection in the evolution of gene duplications. Genome Biol 2002, 3(2):8.1-8.9.

50. Hanada K, Zou C, Lehti-Shiu MD, Shinozaki K, Shiu SH: Importance of lineage-specific expansion of plant tandem duplicates in the adaptive response to environmental stimuli. Plant physiology 2008, 148(2):993-1003.

51. Koch AL: Evolution of antibiotic resistance gene function. Microbiol Rev 1981, 45(2):355-378

52. Martinez $J$ : Antibiotics and antibiotic resistance genes in natural environments. Science 2008, 321(5887):365-367.

53. Widholm JM, Chinnala AR, Ryu JH, Song HS, Eggett T, Brotherton JE: Glyphosate selection of gene amplification in suspension cultures of 3 plant species. Physiol Plantarum 2001, 112(4):540-545.

54. Guillemaud T, Raymond M, Tsagkarakou A, Bernard C, Rochard P, Pasteur N: Quantitative variation and selection of esterase gene amplification in Culex pipiens. Heredity (Edinb) 1999, 83(Pt 1):87-99.

55. Tabashnik BE: Implications of gene amplification for evolution and management of insecticide resistance. J Econ Entomol 1990, 83(4):1170-1176.

56. Gottesman MM, Hrycyna CA, Schoenlein PV, Germann UA, Pastan I: Genetic analysis of the multidrug transporter. Annu Rev Genet 1995, 29:607-649.

57. Chen L, DeVries AL, Cheng CH: Evolution of antifreeze glycoprotein gene from a trypsinogen gene in Antarctic notothenioid fish. Proc Natl Acad SC US A 1997, 94(8):3811-3816.

58. Riehle MM, Bennett AF, Long AD: Genetic architecture of thermal adaptation in Escherichia coli. Proc Natl Acad Sci USA 2001, 98(2):525-530

59. Sonti RV, Roth JR: Role of gene duplications in the adaptation of Salmonella typhimurium to growth on limiting carbon sources. Genetics 1989, 123(1):19-28.

60. Brown CJ, Todd KM, Rosenzweig RF: Multiple duplications of yeast hexose transport genes in response to selection in a glucose-limited environment. Mol Biol Evol 1998, 15(8):931-942.

61. Lai CY, Baumann L, Baumann P: Amplification of trpEG: adaptation of Buchnera aphidicola to an endosymbiotic association with aphids. Proc Natl Acad Sci U S A 1994, 91(9):3819-3823

62. Tang $H$, Kambris $Z$, Lemaitre $B$, Hashimoto $C$ : Two proteases defining a melanization cascade in the immune system of Drosophila. J Biol Chem 2006, 281(38):28097-28104.

63. Levashina EA, Langley E, Green C, Gubb D, Ashburner M, Hoffmann JA, Reichhart JM: Constitutive activation of toll-mediated antifungal defense in serpin-deficient Drosophila. Science 1999, 285(5435):1917-1919.

64. Gorman MJ, Paskewitz SM: Serine proteases as mediators of mosquito immune responses. Insect Biochem Molec 2001, 31(3):257-262.

65. Li L, Memon S, Fan Y, Yang S, Tan S: Recent duplications drive rapid diversification of trypsin genes in 12 Drosophila. Genetica 2012, 140(7-9):297-305.

66. Wu DD, Wang GD, Irwin DM, Zhang YP: A profound role for the expansion of trypsin-like serine protease family in the evolution of hematophagy in mosquito. Mol Biol Evol 2009, 26(10):2333-2341.

67. Zhang J, Zhang YP, Rosenberg HF: Adaptive evolution of a duplicated pancreatic ribonuclease gene in a leaf-eating monkey. Nat Genet 2002 30(4):411-415

68. Werck-Reichhart D, Feyereisen R: Cytochromes P450: a success story. Genome Biol 2000, 1(6):3003.1

69. Guengerich FP: Common and uncommon cytochrome P450 reactions related to metabolism and chemical toxicity. Chem Res Toxicol 2001, 14(6):611-650. 
70. Omiecinski CJ, Remmel RP, Hosagrahara VP: Concise review of the cytochrome P450s and their roles in toxicology. Toxicol Sci 1999, 48(2):151-156

71. Wilson TG: Cyromazine toxicity to Drosophila melanogaster (Diptera: Drosophilidae) and lack of cross-resistance in natural population strains. J Econ Entomol 1997, 90(5):1163-1169.

72. Wilson TG: Genetic evidence that mutants of the methoprene-tolerant gene of Drosophila melanogaster are null mutants. Arch Insect Biochem 1996, 32(3-4):641-649.

73. da Fonseca RR, Antunes A, Melo A, Ramos MJ: Structural divergence and adaptive evolution in mammalian cytochromes P450 2C. Gene 2007 387(1-2):58-66.

74. Tibbles $L$, Woodgett J: The stress-activated protein kinase pathways. Cell Mol Life Sci 1999, 55(10):1230-1254.

75. Sato S, Sanjo H, Takeda K, Ninomiya-Tsuji J, Yamamoto M, Kawai T, Matsumoto K, Takeuchi O, Akira S: Essential function for the kinase TAK1 in innate and adaptive immune responses. Nat Immunol 2005 6(11):1087-1095.

76. Miller MK, Bang ML, Witt CC, Labeit D, Trombitas C, Watanabe K, Granzier H, McElhinny AS, Gregorio CC, Labeit S: The muscle ankyrin repeat proteins: CARP, ankrd2/Arpp and DARP as a family of titin filament-based stress response molecules. J Mol Biol 2003, 333(5):951-964.

77. Yan J, Wang J, Zhang H: An ankyrin repeat-containing protein plays a role in both disease resistance and antioxidation metabolism. Plant $J$ 2002, 29(2):193-202.

78. Schlenke TA, Begun DJ: Natural selection drives Drosophila immune system evolution. Genetics 2003, 164(4):1471-1480.

79. Maroni G, Wise J, Young J, Otto E: Metallothionein gene duplications and metal tolerance in natural populations of Drosophila melanogaster. Genetics 1987, 117(4):739-744.

80. Otto E, Young JE, Maroni G: Structure and expression of a tandem duplication of the Drosophila metallothionein gene. Proc Natl Acad Sci USA 1986, 83(16):6025-6029.

81. Findlay GD, Yi X, Maccoss MJ, Swanson WJ: Proteomics reveals novel Drosophila seminal fluid proteins transferred at mating. PLOS Bio/ 2008, 6(7):e178.

82. Makino T, Kawata M: Habitat variability correlates with duplicate content of Drosophila genomes. Mol Biol Evol 2012, 29(10):3169-3179.

83. Weaver BAA, Cleveland DW: Decoding the links between mitosis, cancer and chemotherapy: The mitotic checkpoint, adaptation, and cell death. Cancer Cell 2005, 8(1):7-12.

84. Lumeng C, Phelps S, Crawford GE, Walden PD, Barald K, Chamberlain JS: Interactions between beta 2-syntrophin and a family of microtubuleassociated serine/threonine kinases. Nat Neurosci 1999, 2(7):611-617.

85. Valiente M, Andres-Pons A, Gomar B, Torres J, Gil A, Tapparel C, Antonarakis SE, Pulido R: Binding of PTEN to specific PDZ domains contributes to PTEN protein stability and phosphorylation by microtubule-associated serine/threonine kinases. J Biol Chem 2005, 280(32):28936-28943.

86. Ekengren S, Hultmark D: A family of Turandot-related genes in the humoral stress response of Drosophila. Biochem Bioph Res Co 2001, 284(4):998-1003.

87. Ekengren S, Tryselius Y, Dushay MS, Liu G, Steiner H, Hultmark D: A humoral stress response in Drosophila. Curr Biol 2001, 11(9):714-718.

88. Frazier AE, Dudek J, Guiard B, Voos W, Li Y, Lind M, Meisinger C, Geissler A, Sickmann A, Meyer HE: Pam16 has an essential role in the mitochondrial protein import motor. Nat Struct Mol Biol 2004, 11(3):226-233.

89. Kelley WL: The J-domain family and the recruitment of chaperone power. Trends Biochem Sci 1998, 23(6):222-227.

90. Qin W, Neal SJ, Robertson RM, Westwood JT, Walker VK: Cold hardening and transcriptional change in Drosophila melanogaster. Insect Mol Biol 2005, 14(6):607-613.

91. Nurminsky DI, Nurminskaya MV, De Aguiar D, Hartl DL: Selective sweep of a newly evolved sperm-specific gene in Drosophila. Nature 1998, 396(6711):572-575

92. Inomata N, Yamazaki T: Adaptive evolution at the molecular level of the duplicatedAmy gene system inDrosophila. J Genet 1996, 75(1):125-137.

93. McQuilton P, St Pierre SE, Thurmond J: FlyBase 101--the basics of navigating FlyBase. Nucleic acids res 2012, 40(Database issue):D706-D714.

94. Larkin MA, Blackshields G, Brown NP, Chenna R, McGettigan PA, McWilliam $\mathrm{H}$, Valentin F, Wallace IM, Wilm A, Lopez R, et al: Clustal W and Clustal X version 2.0. Bioinformatics 2007, 23(21):2947-2948.
95. Tamura K, Peterson D, Peterson N, Stecher G, Nei M, Kumar S: MEGA5: molecular evolutionary genetics analysis using maximum likelihood, evolutionary distance, and maximum parsimony methods. Mol Biol Evol 2011, 28(10):2731-2739.

96. Saitou N, Nei M: The neighbor-joining method: a new method for reconstructing phylogenetic trees. Mol Biol Evol 1987, 4(4):406-425.

97. Punta M, Coggill PC, Eberhardt RY, Mistry J, Tate J, Boursnell C, Pang N, Forslund $\mathrm{K}$, Ceric G, Clements J: The Pfam protein families database. Nucleic Acids Res 2012, 40(D1):D290-D301.

doi:10.1186/1471-2164-14-555

Cite this article as: Zhong et al:: Functional requirements driving the gene duplication in 12 Drosophila species. BMC Genomics 2013 14:555.

\section{Submit your next manuscript to BioMed Central and take full advantage of:}

- Convenient online submission

- Thorough peer review

- No space constraints or color figure charges

- Immediate publication on acceptance

- Inclusion in PubMed, CAS, Scopus and Google Scholar

- Research which is freely available for redistribution

Submit your manuscript at www.biomedcentral.com/submit
C) Biomed Central 